\title{
LARGE GROUPS OF SYMMETRIES OF HANDLEBODIES
}

\author{
A. MILLER AND B. ZIMMERMANN
}

(Communicated by Frederick R. Cohen)

\begin{abstract}
Let $V_{g}$ be an orientable three-dimensional handlebody with genus $g>1$. Let $N(g)$ be the largest order among all finite groups which act effectively on $V_{g}$ and preserve orientation. We show that $4(g+1) \leq N(g) \leq$ $12(g-1)$, and that $N(g)$ equals either $8(q-1)$ or $12(g-1)$ when $g$ is odd. Moreover each of the indicated upper and lower bounds are achieved for infinitely many genera $g$. The techniques which are used lead to more detailed results and also specialize to yield similar results for compact surfaces with nonempty boundary.
\end{abstract}

\section{INTRODUCTION}

A well-known result of Hurwitz states that an orientation preserving symmetry group of a closed Riemann surface of genus $g>1$ has order at most $84(g-1)$ (cf. $\S 4.14$ of [ZVC]). However for fixed values of $g$ the precise determination of the maximal order of such a symmetry group is now recognized as an extremely intricate problem entailing an understanding of the indices of torsion-free normal subgroups of certain triangle groups. In a positive direction it was shown independently by Accola $[\mathrm{A}]$ and by Maclachlan $\left[\mathrm{M}_{1}\right]$ that this maximal order is bounded below by $8(g+1)$ and above by $84(g-1)$, and that each of these bounds is attained for infinitely many $g$ 's. For some classes of symmetry groups more satisfactory results are possible. For instance Wiman (cf. $[\mathrm{H}]$ ) proved that the order of the largest cyclic group acting on a genus $g$ Riemann surface is $2(2 g+1)$. Also the order of the largest abelian symmetry group is $4(g+1)\left[M_{1}\right]$, and the largest nilpotent symmetry group has order at most $16(g-1)\left[Z_{3}\right]$ (related results are derived in $\left[Z_{4}\right]$ ). In this note we will establish similar types of bounds for large groups of symmetries of handlebodies. In this connection it is a general principle that most of the largest group actions on a closed Riemann surface do not extend to a handlebody.

Received by the editors March 28, 1988 and, in revised form, October 10, 1988. The first author was supported in part by a research grant from the University of Oklahoma.

1980 Mathematics Subject Classification (1985 Revision). Primary 57M99; Secondary 57S25, $57 \mathrm{~S} 30$.

Key words and phrases. 3-dimensional handlebody, finite group action, graph of groups, compact 2-manifold with boundary. 
Let $V_{g}$ be an orientable three-dimensional handlebody of genus $g>1$ and let $G$ be a finite group. We consider $G$-actions on $V_{g}$, which are embeddings of $G$ into the group $\operatorname{Diff}^{+}\left(V_{g}\right)$ of orientation preserving diffeomorphisms of $V_{g}$. Let $N(g)$ be the largest order among all finite groups which act on $V_{g}$. In $\left[\mathrm{Z}_{1}, \mathrm{Z}_{2}, \mathrm{MMZ}_{1}\right]$ it was shown that $N(g) \leq 12(g-1)$. We will prove in Theorem 1 that $4(g+1) \leq N(g) \leq 12(g-1)$ and that the lower and upper bounds are each attained for infinitely many $g$ 's. In Theorem 2 we improve this result for odd values of $g$, showing that in this case $8(g-1) \leq N(g) \leq 12(g-1)$ where again the bounds are achieved for infinitely many $g$ 's. Our approach will lead to an even more precise delineation of the problem of computing $N(g)$, as is indicated in $\S 2$ in the Remarks following Theorems 1 and 2 . We will show that for each $g$ there is an integer $n=n_{g}$ with $3 \leq n \leq g+1$ (but $n_{g} \leq 4$ when $g$ is odd) so that $N(g)=[4 n /(n-2)](g-1)$. Infinitely many values of $n$ arise as $n_{g}$ for some genus $g$ but also infinitely many values of $n$ do not arise in this way. Furthermore we will show that if $n$ occurs as $n_{g}$ for one $g$ then it occurs as $n_{g}$ for infinitely many $g$ 's.

For special classes of groups there are more satisfactory results, as in the Riemann surface case. For instance it is shown in Theorem 7.3(c) of $\left[\mathrm{MMZ}_{1}\right]$ that the order of the largest cyclic group acting on the handlebody $V_{g}$ is $2 g$, when $g$ is odd, and $2(g+1)$, when $g$ is even. From our proof of Theorem 1 in $\S 2$ it is evident that $V_{g}$ always admits an action by the abelian group $\mathbf{Z}_{2} \times \mathbf{Z}_{\mathbf{g}+1}$ of order $(2 g+1)$, and an application of Theorem 7.6 of $\left[\mathrm{MMZ}_{1}\right]$ shows that this is the largest possible symmetry of an abelian group on $V_{g}$ except when $g=5$; the handlebody $V_{5}$ admits the group $\mathbf{Z}_{2} \times \mathbf{Z}_{2} \times \mathbf{Z}_{2} \times \mathbf{Z}_{2}^{g}$ as an abelian symmetry of largest possible order. Utilizing Proposition 2 of $\S 1$ it can be shown that the largest nilpotent group acting on $V_{g}$ has order at most $8(g-1)$, and this upper bound is achieved whenever $g-1$ is a power of 2 (as the group constructed in Theorem 2 of $\S 2$ is nilpotent in this case). We also mention that $N(g)=12(g-1)$ for $g=2,3$, or 4 (cf. Theorem 8.2 of $\left[\mathrm{MMZ}_{1}\right]$ ).

In $\S 3$ we will show that our results on large symmetry groups of handlebodies specialize to yield all of the directly analagous results for compact surfaces with nonempty boundary with respect to the concept of algebraic genus. In particular we recover results of May $\left[\mathrm{M}_{2}\right]$.

\section{Preliminaries}

Consider a finite graph of finite groups $(\Gamma, \mathscr{G})$. If $G$ is a finite group then a homomorphism $\rho: \pi_{1}(\Gamma, \mathscr{G}) \rightarrow G$ is said to be finite-injective provided that $\rho$ is one-to-one on each finite subgroup; equivalently, $\rho$ is injective on each vertex group of $\pi_{1}(\Gamma, \mathscr{G})$. In $\left[\mathrm{MMZ}_{1}\right]$ a set of normalized conditions for graphs of groups is given which is used to study group actions on handlebodies. We say that $(\Gamma, \mathscr{G})$ is $G$-admissible if it satisfies the normalized conditions and there is a finite-injective epimorphism from $\pi_{1}(\Gamma, \mathscr{G})$ to $G$. 
Proposition 1 (Theorem $\left.6.1\left[\mathrm{MMZ}_{1}\right]\right)$. A finite group $G$ acts on $V_{g}$ if and only if there is a G-admissible graph of groups $(\Gamma, \mathscr{G})$ with $g-1=-\chi(\Gamma, \mathscr{G})|G|$.

Here $\chi(\Gamma, \mathscr{G})$ denotes the Euler characteristic of $(\Gamma, \mathscr{G})$ which is defined as

$$
\sum \frac{1}{\left|G_{v}\right|}-\sum \frac{1}{\left|G_{e}\right|}
$$

where the sums are taken over all vertex groups $G_{v}$ and over all edge groups $G_{e}$ for unoriented edges $e$. For example the graph of groups

$$
\Gamma(H, C, K)=\frac{C}{H \quad K^{\bullet}}
$$

has $\chi(\Gamma(H, C, K))=1 /|H|+1 /|K|-1 /|C|$ and $\pi_{1}(\Gamma, \mathscr{G})=H{ }^{*} C$. In our setting we will only be interested in graphs of groups $(\Gamma, \mathscr{G})$ with $\chi(\Gamma, \mathscr{G}) \geq$ $-\frac{1}{4}$; the relevant graphs are given in the next Proposition. We use the notation $\Gamma_{n}=\Gamma\left(\mathbf{D}_{2}, \mathbf{Z}_{2}, \mathbf{D}_{\mathbf{n}}\right)$ where $\mathbf{D}_{\mathbf{n}}=\mathbf{Z}_{\mathbf{n}} \circ \mathbf{Z}_{2}$ is the dihedral group of order $2 n$ (the semidirect product of $\mathbf{Z}_{n}$ with $\mathbf{Z}_{2}$ ) and the $\mathbf{Z}_{2}$ edge injects to an order two subgroup not contained in $Z_{n} \circ 1$. (Any two such graphs of groups have isomorphic fundamental groups.)

Proposition 2 (Theorem $\left.7.1\left[\mathrm{MMZ}_{1}\right]\right)$. If $(\Gamma, \mathscr{G})$ is a graph of groups satisfying the normalized conditions and having $0>\chi(\Gamma, \mathscr{G})>-\frac{1}{4}$ then $(\Gamma, \mathscr{G})$ is either $\Gamma_{n}$ for some $n \geq 3$ or one of the following 17. graphs

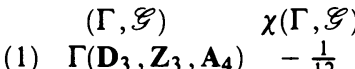

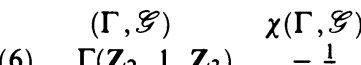
$(\Gamma, \mathscr{G}) \quad \chi(\Gamma, \mathscr{G})$
(2) $\Gamma\left(\mathbf{D}_{4}, \mathbf{Z}_{4}, \mathbf{S}_{4}\right)-\frac{1}{12}$
(7) $\Gamma\left(\mathbf{D}_{2}, \mathbf{Z}_{2}, \mathbf{A}_{4}\right)-\frac{1}{6}$
(3) $\Gamma\left(\mathbf{D}_{5}, \mathbf{Z}_{5}, \mathbf{A}_{5}\right)-\frac{1}{12}$
(8) $\Gamma\left(\mathbf{D}_{3}, \mathbf{Z}_{2}, \mathbf{D}_{3}\right)-\frac{1}{6}$
(4) $\Gamma\left(\mathbf{D}_{3}, \mathbf{Z}_{3}, \mathbf{S}_{4}\right)$
(9) $\Gamma\left(\mathbf{A}_{4}, \mathbf{Z}_{3}, \mathbf{A}_{4}\right)-\frac{1}{6}$
(5) $\Gamma\left(\mathbf{D}_{3}, \mathbf{Z}_{3}, \mathbf{A}_{5}\right)-\frac{3}{20}$
(10) $\Gamma\left(\mathbf{S}_{4}, \mathbf{Z}_{4}, \mathbf{S}_{\mathbf{4}}\right)-\frac{1}{6}$
(11) $\Gamma\left(\mathbf{A}_{5}, \mathbf{Z}_{5}, \mathbf{A}_{5}\right)-\frac{1}{6}$
(12) $\Gamma\left(\mathbf{D}_{2}, \mathbf{Z}_{2}, \mathbf{S}_{4}\right)-\frac{5}{24}$
(13) $\Gamma\left(\mathbf{D}_{3}, \mathbf{Z}_{2}, \mathbf{D}_{4}\right)-\frac{5}{24}$
(14) $\Gamma\left(\mathbf{A}_{4}, \mathbf{Z}_{3}, \mathbf{S}_{4}\right)-\frac{5}{24}$
(15) $\Gamma\left(\mathbf{D}_{2}, \mathbf{Z}_{2}, \mathbf{A}_{5}\right)-\frac{7}{30}$
(16) $\Gamma\left(\mathbf{D}_{3}, \mathbf{Z}_{2}, \mathbf{D}_{5}\right)-\frac{7}{30}$
(17) $\Gamma\left(\mathbf{A}_{4}, \mathbf{Z}_{3}, \mathbf{A}_{5}\right)-\frac{7}{30}$

\section{LARGE SYMMETRIES ON HANDLEBODIES}

We now have the necessary preliminaries to begin our description of $N(g)$. In the subsequent discussions we shall consider the set

$$
\mathscr{F}=\left\{g \in Z^{+} \mid g>12, g-1 \text { is a prime, and } g \not \equiv 2(\bmod 3)\right\} .
$$

Theorem 1. For each integer $g>1$ we have $N(g) \geq 4(g+1)$, and for the infinitely many values of $g$ in $\mathscr{F}, N(g)=4(g+1)$.

Proof. For any integer $g$ let $G=\mathbf{Z}_{2} \times \mathbf{D}_{\mathbf{g}+1}$. There is a finite-injective epimorphism $\rho$ from $\pi_{1}\left(\Gamma_{g+1}\right)=\mathbf{D}_{2} * \mathbf{z}_{2} \mathbf{D}_{\mathbf{g}+1}$ to $G$ with $\rho\left(\mathbf{D}_{\mathbf{g}+1}\right)=1 \times D_{g+1}$ and $\rho\left(\mathbf{D}_{2}\right)=\mathbf{Z}_{2} \times \mathbf{Z}_{2}$. By Proposition 1 it follows that $G$ acts on the handlebody of genus $-\chi\left(\Gamma_{g+1}\right)|G|+1=[(g-1) / 4(g+1)] 4(g+1)+1=g$. We conclude that $N(g) \geq|G|=4(g+1)$.

To prove the second part of the Theorem, suppose that $g \in \mathscr{F}$ and that $V_{g}$ admits a $G$-action for some finite groups $G$ with $|G|>4(g+1)$. By Proposition 1 there is a $G$-admissible graph of groups $(\Gamma, \mathscr{G})$ with $\chi(\Gamma, \mathscr{G})=-(g-1) /|G|$. 
As $-(g-1) /|G|>-(g-1) / 4(g+1)>-\frac{1}{4},(\Gamma, \mathscr{G})$ must be one of the graphs listed in Proposition 2. Let $\rho: \pi_{1}(\Gamma, \mathscr{G}) \rightarrow G$ be a finite-injective epimorphism. We first consider the graphs $(\Gamma, \mathscr{G})=\Gamma_{n}$ which have $\pi_{1}(\Gamma, \mathscr{G})=\mathbf{D}_{2}{ }^{*} \mathbf{z}_{2} \mathbf{D}_{\mathbf{n}}$. The Euler characteristic is $\chi(\Gamma, \mathscr{G})=-(n-2) / 4 n$ and using this we write

$$
g-1=\frac{n-2}{4 n}|G|=\frac{n-2}{d} \frac{d|G|}{4 n}
$$

where $d=1$ if $n$ is odd, and $d=2$ if $n$ is even. As $g-1$ is a prime integer and $2 n$ divides $|G|$ ( $G$ contains a subgroup $\rho\left(\mathbf{D}_{\mathbf{n}}\right)$ with order $2 n$ ), both $d|G| / 4 n$ as well as $(n-2) / d$ are integers and one of them must equal 1 . If $d|G| / 4 n=1$ then $g-1=(n-2) / d$ and

$$
4(g+1)=4\left(\frac{n-2}{d}+2\right)=\frac{4 n+8(d-1)}{d} \geq \frac{4 n}{d}=|G|
$$

which contradicts the choice of $G$. Therefore we must have $(n-2) / d=1$ implying that $n=3$ or $n=4$. We will investigate these possibilities in Cases $A$ and $C$ below, but next we consider the 17 graphs $(\Gamma, \mathscr{G}) \neq \Gamma_{n}$ which are listed in Proposition 2. Suppose $\chi(\Gamma, \mathscr{G})=-A / B$ where $A$ and $B$ are relatively prime integers; then $A \leq 7$ and

$$
g-1=-\chi(\Gamma, \mathscr{G})|G|=\frac{A}{B}|G| .
$$

If $A \neq 1$ then using the primality of $g-1$ we have $|G|=B$ and $g-1=A \leq 7$. This contradicts the fact that $g>12$. Thus it must be that $A=1$. Referring to Proposition 2 we see that $|G|=B(g-1)$ where $B$ is 6,8 or 12 , and the graphs (5) and (12)-(17) are ruled out. Since $g-1$ is a large prime, $G$ cannot contain subgroups with order 60 or 24 (such as $\mathbf{A}_{5}$ or $\mathbf{S}_{4}$ ). Therefore $(\Gamma, \mathscr{G})$ cannot be any of the graphs (2), (3), (4), (10), or (11) from Proposition 2. Also the graphs (7) and (9) are ruled out since here $|G|=6(g-1)$ and $G$ cannot contain an order 12 subgroup (such as $\mathbf{A}_{4}$ ). This leaves the graphs (1), (6) and (8), as well as $\Gamma_{3}$ and $\Gamma_{4}$ which must be considered individually.

Case A. $(\Gamma, \mathscr{G})=\Gamma_{3}$. Then $|G|=12(g-1)$ and $\pi_{1}(\Gamma, \mathscr{G})=\mathbf{D}_{2} * \mathbf{z}_{2} \mathbf{D}_{3}$. A Sylow $(g-1)$-subgroup $P$ of $G$ is cyclic with order $g-1$ and by Sylow's Theorem the number of such subgroups divides 12 and is congruent to 1 mod $(g-1)$. Since $g-1 \geq 12$ it follows that $P$ is normal in $G$. The well-known Schur-Zassenhaus Theorem states that in a finite group any normal subgroup has a complement if its order is relatively prime to its index. In our setting this implies that $P$ must have a complement, i.e. $-G$ is a semidirect product $P \circ \bar{G}$ where $\bar{G}$ has order 12 . (Our usage of this Theorem can be avoided by elementary arguments but we include it here, and in the sequel, for the sake of expediency.) Under the projection from $G$ to $\bar{G}$ the image of $\rho\left(\mathbf{D}_{3}\right)$ is a $\mathbf{D}_{3}$ subgroup and hence $\bar{G} \cong \mathbf{D}_{6}$ as $\mathbf{D}_{6}$ is the only group of order 12 which contains such a subgroup. Now $\operatorname{Aut}(P)$ is cyclic (in fact $\cong \mathbf{Z}_{\mathbf{g}-2}$ ) and the action of $\bar{G}$ on $P$ must factor through $\left(\mathbf{D}_{6}\right)_{a b} \cong \mathbf{D}_{2}$. It follows that the unique $\mathbf{Z}_{3}$ subgroup $H$ of $\bar{G}$ acts trivially on $P$ and as a result $H$ is the only order 3 subgroup of 
$G$. (If $(x y)^{3}=1$ where $x \in P$ and $y \in G$ then $y^{3}=1$ implying that $x^{3}$ and then $x$ are trivial.) The image of $\rho$ is generated by $\rho\left(\mathbf{D}_{2}\right)$ together with $H$; as $H$ is normal this image has order 12 so that $\rho$ is not surjective. From this contradiction we conclude that Case A cannot arise.

Case B. $(\Gamma, \mathscr{G})=\Gamma\left(\mathbf{D}_{3}, \mathbf{Z}_{3}, \mathbf{A}_{4}\right)$. Then $|G|=12(g-1)$ and $\pi_{1}(\Gamma, \mathscr{G})=$ $\mathbf{D}_{3}{ }^{*} \mathbf{z}_{3} \mathbf{A}_{4}$. Let $P$ be a Sylow $(g-1)$-subgroup of $G$. As before the Sylow theory implies that $P$ is normal in $G$. Since $\rho\left(\mathbf{A}_{4}\right)$ has order 12 it follows that $G=P \circ \rho\left(\mathbf{A}_{4}\right)$. The $\rho\left(\mathbf{A}_{4}\right)$ action on $P$ factors through $\left(\mathbf{A}_{4}\right)_{a b} \cong \mathbf{Z}_{3}$ so every order 2 element of $\rho\left(\mathbf{A}_{4}\right)$ commutes with $P$. If $x y\left(x \in P, y \in \rho\left(\mathbf{A}_{4}\right)\right)$ is an element of $G$ with order two then $y^{2}=1$ and $1=(x y)^{2}=x^{2}$ so that $x=1$. This shows that every order two element is contained in $\rho\left(\mathbf{A}_{4}\right)$. In particular it follows that $\rho\left(\mathbf{D}_{3}\right) \subset \rho\left(\mathbf{A}_{4}\right)$ which is impossible as $\mathbf{A}_{4}$ has no $\mathbf{D}_{3}$ subgroup.

Case C. $(\Gamma, \mathscr{G})=\Gamma_{4}$. Then $|G|=8(g-1)$ and $\pi_{1}(\Gamma, \mathscr{G})=\mathbf{D}_{2} * \mathbf{z}_{2} \mathbf{D}_{4}$. Again we have a cyclic normal Sylow subgroup $P$ of $G$ with order $g-1$. Since $\left|\rho\left(\mathbf{D}_{4}\right)\right|=8$ we must have $G=P \circ_{\psi} \rho\left(\mathbf{D}_{4}\right)$ where $\psi: \rho\left(\mathbf{D}_{4}\right) \rightarrow \operatorname{Aut}(P)$ is the semidirect product action. Choose elements $a \in \rho\left(\mathbf{D}_{4}\right)$ and $b \in \rho\left(\mathbf{D}_{4}\right) \cap \rho\left(\mathbf{D}_{2}\right)$ so that $a^{4}=b^{2}=1$ and $b a b^{-1}=a^{-1}$. Suppose that $\rho\left(\mathbf{D}_{2}\right)$ is the subgroup $\langle b, x y\rangle$ generated by $b$ and $x y$ where $x \in P$ and $y \in \rho\left(\mathbf{D}_{4}\right)$. Then $x y=$ $b(x y) b^{-1}=\psi(b)(x) b y b^{-1}$, which implies that $\psi(b)(x)=x$ and $b y b^{-1}=y$. This shows that $y$ is an element of the centralizer $\left\langle b, a^{2}\right\rangle$ of $b$ in $\rho\left(\mathbf{D}_{4}\right)$, and that if $x \neq 1$ then $b \in \operatorname{ker}(\psi)$. Since $a^{2} \in \operatorname{ker}(\psi)$ it follows that either $y \in \operatorname{ker}(\psi)$ or $x=1$. But $x y$ has order two so $1=(x y)^{2}=x^{2} y^{2}$ and it must be that $x=1$. Therefore $\rho\left(\mathbf{D}_{2}\right)=\langle b, y\rangle \subset \rho\left(\mathbf{D}_{4}\right)$ which contradicts the fact that $\rho$ is surjective.

Case D. $(\Gamma, \mathscr{G})=\Gamma\left(\mathbf{Z}_{2}, 1, \mathbf{Z}_{3}\right)$. Then $|G|=6(g-1)$ and $\pi_{1}(\Gamma, \mathscr{G})=\mathbf{Z}_{2} * \mathbf{Z}_{3}$. Applying the Sylow theory as before, we see that $G$ contains a normal cyclic subgroup $P$ of order $g-1$. By the Schur-Zassenhaus Theorem, $G=P \circ \bar{G}$ where $\bar{G}$ has order 6 . Since $\operatorname{Aut}(P) \cong \mathbf{Z}_{\mathbf{g}-2}$ and $g-2 \not \equiv 0(\bmod 3)$ each order three element of $G$ acts trivially on $P$. Therefore $\rho\left(Z_{3}\right)$ is normal in $G$ and the image of $\rho$ equals $\left\langle\rho\left(\mathbf{Z}_{3}\right), \rho\left(\mathbf{Z}_{2}\right)\right\rangle$ which has order 6 . This contradicts the fact that $\rho$ is surjective.

Case E. $(\Gamma, \mathscr{G})=\Gamma\left(\mathbf{D}_{3}, \mathbf{Z}_{2}, \mathbf{D}_{3}\right)$. Then $|G|=6(g-1)$ and $\pi_{1}(\Gamma, \mathscr{G})=$ $\mathbf{D}_{3}{ }^{*} \mathbf{z}_{2} \mathbf{D}_{3}$. Again we have $G=P \circ \rho\left(\mathbf{D}_{3}\right)$ where $P$ is cyclic of order $g-1$. Since $\left(\mathbf{D}_{3}\right)_{a b} \cong \mathbf{Z}_{2}$ each element of order three in $\rho\left(\mathbf{D}_{3}\right)$ acts trivailly on $P$. It follows that each element of order three is contained in $\rho\left(\mathbf{D}_{3}\right)$ and that $\rho\left(\mathbf{D}_{3}\right)$ is the image of $\rho$, contradicting the fact that $\rho$ is surjective.

We have now shown that if $g \in \mathscr{F}$ then $V_{g}$ admits no $G$-action where $|G|>4(g+1)$ and the proof of the Theorem is complete.

Remarks (1). The Euler characteristic of $\Gamma_{n}$ is $-(n-2) / 4 n$ and a quick glance at the 17 cases listed in Proposition 2 shows that all graphs of groups $(\Gamma, \mathscr{G})$ with $0>\chi(\Gamma, \mathscr{G})>-\frac{1}{4}$ have $\chi(\Gamma, \mathscr{G})=-(n-2) / 4 n$ for some $n \geq 3$. It 
follows from Theorem 1 that for every $g>1$ we have $N(g)=[4 n /(n-2)(g-1)$ for some $n=n_{g}$ with $3 \leq n \leq g+1$, and that $n_{g}=g+1$ when $g \in \mathscr{F}$.

(2) In the proof of Theorem 1, it was only in Case D that we used the property that $g \not \equiv 2(\bmod 3)$ when $g \in \mathscr{F}$. Consider the complementary set

$$
\mathscr{F}^{\prime}=\left\{g \in \mathbf{Z}^{+} \mid g>12, g-1 \text { is a prime, and } g \equiv 2(\bmod 3)\right\} \text {. }
$$

If $g \in \mathscr{F}^{\prime}$ then the group $G=\mathbf{Z}_{\mathbf{g}-1} \circ_{\psi} \mathbf{Z}_{6}$ whose action $\psi: \mathbf{Z}_{6} \rightarrow \operatorname{Aut}\left(\mathbf{Z}_{\mathbf{g}-1}\right)$ is one-to-one, is easily seen to be a finite-injective quotient of $\mathbf{Z}_{2} * \mathbf{Z}_{3}$. (If $\mathbf{Z}_{\mathbf{g}-1} \circ 1=\langle a\rangle$ and $1 \circ \mathbf{Z}_{6}=\langle t\rangle$ then $G$ is generated by $\left\langle t^{3}\right\rangle \cong \mathbf{Z}_{2}$ together with $\left\langle a t^{2}\right\rangle \cong \mathbf{Z}_{3}$.) This shows that

$$
N(g)=6(g-1) \text { for the infinitely many values of } g \text { in } \mathscr{F}^{\prime} .
$$

In other words, $n_{g}=6$ when $g \in \mathscr{F}^{\prime}$.

Theorem 2. If $g$ is an odd integer then $N(g) \geq 8(g-1)$. For the infinitely many integers of the form $g=2 p+1$ where $p>23$ is prime we have $N(g)=8(g-1)$.

Proof. For each odd integer $g$ we will construct a group

$$
G=\left(\mathbf{Z}_{(\mathbf{g}-1) / 2} \circ_{\phi} \mathbf{D}_{4}\right) \circ_{\psi} \mathbf{Z}_{2}
$$

which is a finite-injective quotient of $\pi_{1}\left(\Gamma_{4}\right)=\mathbf{D}_{2}{ }^{*} \mathbf{z}_{2} \mathbf{D}_{4}$. By Proposition 1 and since $\chi\left(\Gamma_{4}\right)=-\frac{1}{8}$ it follows that $G$ acts on the handlebody of genus $\frac{1}{8}|G|+1=g$ and $N(g) \geq|G|=8(g-1)$. We now describe the actions $\phi$ and $\psi$ used to define $G$. Writing $\mathbf{Z}_{(\mathbf{g}-1) / 2}=\left\langle x \mid x^{(g-1) / 2}=1\right\rangle, \mathbf{D}_{4}=\langle y, z| y^{4}=$ $\left.z^{2}=1, z y z^{-1}=y^{-1}\right\rangle$, and $\mathbf{Z}_{2}=\left\langle t \mid t^{2}=1\right\rangle$, the actions are given by

$$
\phi:\left\{\begin{array}{l}
y x y^{-1}=x^{-1} \\
z x z^{-1}=x
\end{array} \quad \psi:\left\{\begin{array}{l}
t x t^{-1}=x^{-1} \\
t y t^{-1}=x y \\
t z t^{-1}=z
\end{array}\right.\right.
$$

and it is straightforward to check that they are well defined. It remains to show that $\Gamma_{4}$ is $G$-admissible. Write $\mathbf{D}_{2}{ }^{*} \mathrm{z}_{2} \mathbf{D}_{4}=\langle T, Y, Z\rangle$ where $T^{2}=$ $Z^{2}=Y^{4}=1, Z T Z^{-1}=T$, and $Z Y Z^{-1}=Y^{-1}$. Then a homomorphism $\rho: \mathbf{D}_{2}{ }^{*} \mathrm{Z}_{2} \mathbf{D}_{4} \rightarrow G$ is given by $\rho(T)=t, \rho(Y)=y$ and $\rho(Z)=z$. Clearly $\rho$ is finite-injective and it is surjective since $t y t^{-1} y^{-1}=x$. This completes the proof of the first part of the Theorem.

Now suppose that $g=2 p+1$ where $p>23$ is prime, and suppose that $V_{g}$ admits a $G$-action with $|G|>8(g-1)$. Then there is a $G$-admissible graph of groups $(\Gamma, \mathscr{G})$ with $\chi(\Gamma, \mathscr{G})=-(g-1) /|G|>-\frac{1}{8}$. Therefore $\chi(\Gamma, \mathscr{G})=-\frac{1}{12}$, $(\Gamma, \mathscr{G})$ is either $\Gamma_{3}$ or one of the first 2 of the 17 graphs listed in Proposition 2 , and $|G|=12(g-1)=24 p$. As before (since $p>23$ ) a Sylow $p$-subgroup $P$ of $G$ is normal and $G=P \circ_{\phi} \bar{G}$ where $|\bar{G}|=24$. The composition of a finite-injective epimorphism $\rho: \pi_{1}(\Gamma, \mathscr{G}) \rightarrow G$ with the projection $G \rightarrow \bar{G}$ is again a finite-injective epimorphism since no element of $\pi_{1}(\Gamma, \mathscr{G})$ has order 
$p>23$. By Proposition $1, \bar{G}$ acts on the handlebody of genus $\frac{1}{12}|\bar{G}|+1=3$. But by Theorem 8.2(c) of [MMZ $]$ the only group of order 24 acting on $V_{3}$ is $\mathbf{S}_{4}$, so $\bar{G} \cong \mathbf{S}_{4}$. The action $\phi: \bar{G} \rightarrow \operatorname{Aut}(P)$ factors through $\left(\mathbf{S}_{4}\right)_{a b} \cong \mathbf{Z}_{2}$. If $\phi$ is trivial then $G=P \times \bar{G}$ and it readily follows that image $(\rho) \subseteq 1 \times \bar{G}$ contradicting the fact that $\rho$ is surjective. Thus we assume that $\phi$ is nontrivial which implies that $\operatorname{ker}(\phi)$ has index two in $\bar{G}$ and $\operatorname{ker}(\phi) \cong \mathbf{A}_{4}$. We now consider the three possible graphs $(\Gamma, \mathscr{G})$ individually.

Case A. $(\Gamma, \mathscr{G})=\Gamma\left(\mathbf{D}_{4}, \mathbf{Z}_{4}, \mathbf{S}_{4}\right)$ and $\pi_{1}(\Gamma, \mathscr{G})=\mathbf{D}_{4} * \mathbf{z}_{4} \mathbf{S}_{4}$. We may assume that $\rho\left(\mathbf{S}_{4}\right)=1 \circ \bar{G} \subseteq P \circ \bar{G}=G$. Suppose that $\rho\left(\mathbf{D}_{4}\right)$ is generated by $\langle z, x w\rangle$ where $z, w \in \bar{G}, x \in P, z$ has order $4, x w$ has order 2 and $(x w) z(x w)^{-1}=$ $z^{-1}$. But then $z^{-1}=x\left(w z w^{-1}\right) x^{-1}=x^{2}\left(w z w^{-1}\right)$ since $w z w^{-1} \notin \operatorname{ker}(\phi)$ $\left(\mathbf{A}_{4}\right.$ contains no element of order 4). From this it follows that $x^{2}=1$ and $x=1$. Since image $(\rho)=\langle x w, \bar{G}\rangle \subseteq \bar{G}$ this contradicts the hypothesis that $\rho$ is surjective.

Case B. $(\Gamma, \mathscr{G})=\Gamma\left(\mathbf{D}_{3}, \mathbf{Z}_{3}, \mathbf{A}_{4}\right)$ and $\pi_{1}(\Gamma, \mathscr{G})=\mathbf{D}_{3}{ }^{*} \mathbf{z}_{3} \mathbf{A}_{4}$. We first observe that $\rho\left(\mathbf{A}_{4}\right) \subseteq 1 \circ \bar{G}$ since if $x w$ has order 2 or 3 where $x \in P$ and $w \in \operatorname{ker}(\phi)$ then $x=1$ (since either $x^{2}=1$ or $x^{3}=1$ ). Now suppose that $x w \quad(x \in P, w \in \bar{G})$ is an element of order 2 in $\rho\left(\mathbf{D}_{3}-\mathbf{Z}_{3}\right)$. Since $\rho$ is surjective we must have $w \notin \operatorname{ker}(\phi)$. Let $y \in \operatorname{ker}(\phi)$ be an element of order 3 so that $w y$ has order 4 (thus $\bar{G}$ is generated by $\{w, y\}$ ). Then $(x w y)^{2}=x(w y) x(w y)=(w y)^{2}$ so $x w y$ has order 4. This implies that $\{x w, y\}$ generates an $\mathbf{S}_{4}$ subgroup of $G$ and this subgroup contains $\rho\left(\mathbf{A}_{4}\right)$ (which is generated by $\left\{(w y)^{2}, y\right\}$ ). Hence the image of $\rho$, which is generated by $\rho\left(\mathbf{A}_{4}\right)$ together with $x w$, equals this $\mathbf{S}_{4}$ subgroup and $\rho$ is not surjective.

Case C. $(\Gamma, \mathscr{G})=\Gamma_{3}$ and $\pi_{1}(\Gamma, \mathscr{G})=\mathbf{D}_{2}{ }^{*} \mathbf{z}_{2} \mathbf{D}_{3}$. Let $W, Y$, and $Z$ be elements of $\mathbf{D}_{2} * \mathbf{z}_{2} \mathbf{D}_{3}$ so that $\mathbf{D}_{2}=\left\langle W, Y \mid W^{2}=Y^{2}=(W Y)^{2}=1\right\rangle$ and $\mathbf{D}_{3}=\left\langle Y, Z \mid Y^{2}=Z^{3}=1, Y Z Y^{-1}=Z^{-1}\right\rangle$. Let $\rho(W)=x_{1} w, \rho(Y)=x_{2} y$ and $\rho(Z)=x_{3} z$ where $x_{1}, x_{2}, x_{3} \in P$ and $w, y, z \in \bar{G}$. Note that $z \in \operatorname{ker}(\phi)$ ( $\mathbf{A}_{4}$ contains all order 3 element of $\mathbf{S}_{4}$ ), $y \notin \operatorname{ker}(\phi)$ (otherwise $\mathbf{A}_{4}$ would contain a $\mathbf{D}_{3}$ subgroup) and $w \in \operatorname{ker}(\phi)$ (if not then replace $W$ with $W Y$ ). As shown in Case B, we must have $x_{1}=x_{3}=1$ so $\langle\rho(W), \rho(Z)\rangle=1 \circ \operatorname{ker}(\phi)=\mathbf{A}_{4}$. But $\langle\rho(W), \rho(Z)\rangle$ has index 2 in image $(\rho)$, so $\rho$ is not surjective.

Remarks (1). Theorem 2 implies that if $g$ is odd then either $N(g)=8(g-1)$ or $N(g)=12(g-1)$, and that $N(g)=8(g-1)$ for infinitely many odd integers. We now show that also $N(g)=12(g-1)$ for infinitely many odd integers. This was shown in $\left[Z_{2}\right]$ but we will derive some further results from our argument. This type of argument is also employed in [GM].

Let $g>1$ be an integer and suppose that $N(g)=A(g-1)$. (Thus $A=$ $4 n /(n-2)$ where $\left.n=n_{g}.\right)$ Then there is a finite group $G$ with $|G|=N(g)$ and a $G$-admissible graph of groups $(\Gamma, \mathscr{G})$ such that $\chi(\Gamma, \mathscr{G})=-1 / A$. This 
determines a group extension

$$
1 \rightarrow F_{g} \rightarrow \pi_{1}(\Gamma, \mathscr{G}) \rightarrow G \rightarrow 1
$$

where $F_{g}$ is free of rank $g$. For any positive integer $m$ let $K_{m}$ be the kernel of the projection $F_{g} \rightarrow H_{1}\left(F_{g} ; \mathbf{Z}_{\mathrm{m}}\right)$. Then $K_{m}$ is characteristic in $F_{g}$ and normal in $\pi_{1}(\Gamma, \mathscr{G})$. Let $G_{m}=\pi_{1}(\Gamma, \mathscr{G}) / K_{m}$; this is an extension of $G$ by $\mathbf{Z}_{\mathrm{m}}^{g}$ and has order $|G| m^{g}=A(g-1) m^{g}$. Since the projection $\pi_{1}(\Gamma, \mathscr{G}) \rightarrow G_{m}$ is finite-injective, there is an action of $G_{m}$ on the handlebody of genus $g_{m}=$ $-\chi(\Gamma, \mathscr{G})\left|G_{m}\right|+1=(1 / A)|G| m^{g}+1=(g-1) m^{g}+1$. We conclude that, for each positive integer $m$ :

If $N(g)=A(g-1)$ and $g_{m}=(g-1) m^{g}+1$ then $N\left(g_{m}\right) \geq A\left(g_{m}-1\right)$.

In particular, if $N(g)=12(g-1)$ then $N\left(g_{m}\right)=12\left(g_{m}-1\right)$ for each $m$. To illustrate, since $N(g)=12(g-1)$ for $g=2,3$, or 4 it follows that $N\left(g^{\prime}\right)=$ $12\left(g^{\prime}-1\right)$ whenever $g^{\prime}=m^{2}+1,2 m^{3}+1$, or $3 m^{4}+1$ for any positive integer $m$.

(2) Expanding on the previous remark, we show that:

$$
\text { If } n=n_{g} \text { for some } g \text { then } n=n_{g} \text { for infinitely many } g \text { 's. }
$$

Suppose that $n=n_{g}$ for some genus $g$ and let $A=4 n /(n-2)$, so that $N(g)=A(g-1)$. Then $n \geq 3$ and $-1 / A>-\frac{1}{4}$. By Proposition 2 there are only finitely many graphs of groups $(\Gamma, \mathscr{G})$ satisfying the normalized conditions and having $\chi(\Gamma, \mathscr{G}) \geq-1 / A$. Let $p>12(g-1)$ be any prime which does not divide the order of a vertex group of any of these graphs. (Thus $p$ does not divide the numerator or denominator of the reduced rational number form of the Euler characteristic of any of these graphs.) Let $g_{p}=(g-1) p^{g}+1$. By (1) of Remarks, $N\left(g_{p}\right) \geq A\left(g_{p}-1\right)$. Suppose that $N\left(g_{p}\right)>A\left(g_{p}-1\right)$, then there is a finite group $G$ together with graph of groups $(\Gamma, \mathscr{G})$ and a finiteinjective epimorphism $\rho: \pi_{1}(\Gamma, \mathscr{G}) \rightarrow G$ for which $|G|=-\left(g_{p}-1\right) / \chi(\Gamma, \mathscr{G})=$ $-(g-1) p^{g} / \chi(\Gamma, \mathscr{G})$ and $-1 / \chi(\Gamma, \mathscr{G})>A$. Let $P$ be a Sylow $p$-subgroup of $G$. As $p$ divides neither the numerator nor the denominator of $-(g-1) / \chi(\Gamma, \mathscr{G})$, $P$ has order $p^{g}$. Using Sylow's Theorem and the inequalities $p>12(g-1)>$ $-(g-1) / \chi(\Gamma, \mathscr{G}), P$ is normal. The composition of $\rho$ with the projection $G \rightarrow G / P$ gives an epimorphism $\pi_{1}(\Gamma, \mathscr{G}) \rightarrow G / P$ which is finite-injective because no finite subgroup of $\pi_{1}(\Gamma, \mathscr{G})$ has order $p$. It follows that the factor group $G / P$ acts on the handlebody of genus $-\chi(\Gamma, \mathscr{G})|G / P|+1=g$. Also $A(g-1)=N(g) \geq|G / P|=-(g-1) / \chi(\Gamma, \mathscr{G})$ from which we obtain the contradiction $A \geq-1 / \chi(\Gamma, \mathscr{G})$.

(3) We have now seen that for each $g$ the rational number $N(g) /(g-1)$ equals $4 n /(n-2)$ for some $n \geq 3$, and that if $4 n /(n-2)$ occurs once then it occurs for infinitely many genera $g$. On the other hand:

Infinitely many values of $4 n /(n-2)$ do not occur as $N(g) /(g-1)$ for any $g$. 
As an example, if $n \equiv 2(\bmod 16)$ and $n \neq 2$ the values $4 n /(n-2)$ do not arise in this way. To demonstrate this, suppose that $N(g)=4 n(g-1) /(n-2)$ for some $g$ where $n$ satisfies these conditions. Then $(n-2) N(g)=4 n(g-1)$. Since $(n-2) N(g) \equiv 0(\bmod 16)$ and $4 n(g-1) \equiv 8(g-1)(\bmod 16)$ we must have $8(g-1) \equiv 0(\bmod 16)$ or, equivalently, $g-1$ is even. Then Theorem 2 implies that $4 n /(n-2)=N(g) /(g-1)$ is at least 8 which contradicts the hypothesis that $n>2$.

\section{LARGE SYMMETRIES ON BOUNDED SURFACES}

We conclude by showing that the arguments of $\S 2$ readily lead to analogous results for actions on bounded surfaces. A bounded surface is a compact 2manifold with nonempty boundary (either orientable or not) and its algebraic genus is the rank of its fundamental group. Let $N_{2}(g)$ be the maximal order of all finite groups acting on bounded surfaces of algebraic genus $g$ (possibly reversing orientation). To study actions on bounded surfaces it is convenient to use an approach similar to that given in Proposition 1 for actions on handlebodies. There is a set of conditions on $(\Gamma, \mathscr{G})$, which we refer to as the normalized conditions for surface actions, so that a group $G$ acts on some bounded surface with algebraic genus $g$ if and only if $G$ is the finite-injective quotient of $\pi_{1}(\Gamma, \mathscr{G})$ for such a graph. (This result is proved in $\left[\mathrm{MMZ}_{2}\right]$.) The normalized conditions for surface actions imply the normalized conditions (for handlebodies), so that relevant graphs with $0>\chi(\Gamma, \mathscr{G})>-\frac{1}{4}$ may be found in Proposition 2. Explicitly, these graphs are all of the $\Gamma_{n}$ graphs, as well as graphs (6), (8), (13) and (16).

Corollary. (a) $N_{2}(g) \geq 4(g+1)$ and $N_{2}(g)=4(g+1)$ for infinitely many integers $g$.

(b) If $g$ is odd then $N_{2}(g) \geq 8(g-1)$ and $N_{2}(g)=8(g-1)$ for infinitely many odd integers.

Proof. In proving the two analogous inequalities for handlebody actions (Theorems 1 and 2) we used constructions involving the graphs $\Gamma_{g+1}$ and $\Gamma_{4}$. Since these satisfy the normalized conditions for surfaces, the inequalities in (a) and (b) follow. To complete the proof one observes that $N_{2}(g) \leq N(g)$ : if $G$ acts on a bounded surface $S$ with algebraic genus $g$ then $G$ acts on $V_{g}$ by taking a (possibly twisted to make the handlebody orientable) product of $\stackrel{g}{S}$ with $[0,1]$ and extending the group action in the natural way.

The procedure used in proving the Corollary can also be used to show that each of the properties derived in the remarks following Theorems 1 and 2 also holds for actions on bounded surfaces. In fact for all of the genera $g$ we have considered here $N(g)=N_{2}(g)$. It remains open whether or not this equality always holds, although we suspect that it does not. 


\section{REFERENCES}

[A] R. Accola, On the number of automorphisms of a closed Riemann surface, Trans. Amer. Math. Soc. 131 (1968), 398-408.

[GM] N. Greenleaf and C. May, Bordered Klein surfaces with maximal symmetry, Trans. Amer. Math. Soc. 274 (1982), 265-283.

[H] W. J. Harvey, Cyclic groups of automorphisms of a compact Riemann surface, Quart. J. Math. Oxford Ser. (2) 17 (1966), 86-97.

[ $\mathrm{M}_{1}$ ] C. Maclachlan, $A$ bound for the number of automorphisms of a compact Riemann surface, $\mathrm{J}$. London Math. Soc. 44 (1969), 265-272.

[ $\mathrm{M}_{2}$ ] C. May, $A$ bound for the number of automorphisms of a compact Klein surface with boundary, Proc. Amer. Math. Soc. 63 (1977), 273-280.

[ $\mathrm{MMZ}_{1}$ ] D. McCullough, A. Miller, and B. Zimmermann, Group actions on handlebodies, Proc. London Math. Soc. (to appear).

$\left[\mathrm{MMZ}_{2}\right] \_$, Group actions on nonclosed surfaces, 1988, (preprint).

[ZVC] H. Zieschang, E. Vogt, and H-D. Coldewey, Surfaces and planar discontinuous groups, Lecture Notes in Math., vol. 835, Springer-Verlag, Berlin and New York, 1980.

[ $Z_{1}$ ] B. Zimmermann, Uber Abbildungsklassen von Henkelkörpern, Arch. Math. 33 (1979), 379382.

$\left[\mathrm{Z}_{2}\right]$, Uber Homoömorphismen n-dimensionaler Henkelkörper und endliche Erweiterungen von Schottky-Gruppen, Comment. Math. Helv. 56 (1981), 474-486.

[ $\left.Z_{3}\right]$ R. Zomorrodian, Nilpotent automorphism groups of Riemann surfaces, Trans. Amer. Math. Soc. 288 (1985), 241-255.

$\left[\mathrm{Z}_{4}\right]$, Classification of p-groups of automorphisms of Riemann surfaces and their lower central series, Glasgow Math. J. 29 (1987), 237-244.

Department of Mathematics, University of Oklahoma, Norman, Oklahoma 73019

Dipartimento di Scienze Matematiche, Università degli studi di Trieste, 34100 TRIESTE, ITALY 